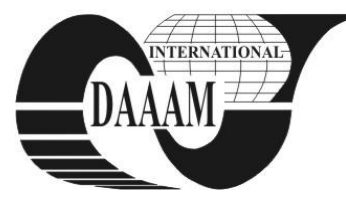

Annals of DAAAM for 2011 \& Proceedings of the 22nd International DAAAM Symposium, Volume 22, No. 1, ISSN 1726-9679 ISBN 978-3-901509-83-4, Editor B. Katalinic, Published by DAAAM International, Vienna, Austria, EU, 2011 Make Harmony between Technology and Nature, and Your Mind will Fly Free as a Bird

\title{
COMPUTING THE TORQUE DEMAND OF A PROSTHETIC LEG
}

\author{
BASER, O[zgun]; KESKIN, O[nur]; CETIN, L[event] \& UYAR, E[rol]
}

\begin{abstract}
Dynamics of a prosthetic leg designed, which is an artificial hybrid leg with a polycentric knee articulation, is derived in this paper to obtain the torque demand of the system. Distinctive feature of the artificial hybrid leg is that it is a serial manipulator, the knee joint of which is designed as a four bar mechanism. The model has two degrees of freedom, one at the ankle, the other at the knee joint. For the knee joint, iterative inverse kinematic analysis is done in order to obtain the poses of all joints of the polycentric mechanism for the given arbitrary trajectory of hip joint. Dynamic torques can be calculated only after kinematical analysis has been achieved, as the external torques will have to be taken into consideration. Finally, torque demands for the motors which are going to actuate the hybrid artificial leg system can be computed obtaining the system dynamics.

Key words: prosthetic leg, artificial hybrid leg, inverse iterative kinematics, dynamic analysis, polycentric knee mechanism
\end{abstract}

\section{INTRODUCTION}

Because of the successes of robotics technology in industry such as precise velocity and force control, rapid response, making of correct self decision, repeating the task without significant errors etc., new challenges to develop applicable and profitable robotic devices for other non-traditional branches of industry were launched in scientific research circles. One such challenge is in the field of medical robotics. It is a subbranch of bio-mechanics, which is an interdisciplinary study of biology, mechanics and electronics, with an aim to incorporate biological organs with electro-mechanical systems.

Design of prosthetic devices as artificial leg having active knee joint is one of the most significant research areas of medicine robotics. Today, there exist single axis knee joints, pioneered by the Otto Bock's with his product C-Leg which has some technological successes discussed in the literature (Pinzur \& Bowker, 1999). Although the benefis of single axis knee joints are introduced in prior studies (Michael, 1999; Fujimoto et al., 1992; Kapti \& Yucenur, 2006), in the studies by Radcliffe it is proved that the amputees can walk with lower torque demands if the knee joint of the artificial leg is designed as a polycentric knee mechanism. Reduction of the joint torques can be achieved by changing the position of the instant centre of rotation of the mechanism, which cannot be done in single axis knee joints (Radcliffe, 1994). Lower torque demand enables the usage of smaller motors with lower motor powers in the artificial leg design. Because of the advantages of polycentric knee joint, dynamic analysis of the artificial leg having this kind of knee articulation is reported in this study.

\section{THEORETICAL MODEL}

The model of artificial leg is considered as an artificial hybrid leg having two degrees of freedom, one at the ankle joint, Joint $O$ and the other at Joint A of the four bar mechanism (Figure 1). By activating the system with motors mounted on these two joints, hip joint can track an arbitrary path which complies with the conditions of natural walking.

In Figure $1, \theta_{a}$ is the angle of the ankle joint, $\theta_{1}, \theta_{2}, \theta_{3}$ and $\theta_{4}$ are the joint variables for the joints $D, A, B$ and $C$ respectively. $\alpha$ and $\beta$ are the geometric constants.

\subsection{Forward Kinematics to Obtain Workspace}

Forward kinematics can be defined as the computation of the position and orientation of robot's end effector as a function of joint angles. The workspace of artificial leg can be derived by this method.

Kinematic equation for the close chain $\mathrm{ABCD}$ shown in Figure 1 is given as in Eq.(1). Here, $l_{2}, l_{3}, l_{4}$ and $l_{1}$ are the lengths of the links $A B, B C, C D$ and $D A$, respectively. Note that $\theta_{1}$ is related with variable $\theta_{a}$ by geometric relations. In Eq.(1), $\theta_{a}$ and $\theta_{2}$ are known variables and all other variables of the four bar mechanism can be computed.

As for the position of hip joint, the following open chain equation can be used:

$$
\begin{gathered}
l_{2} \cdot e^{i\left(\theta_{a}+\theta_{2}\right)}+l_{3} \cdot e^{i \theta_{3}}-l_{4} \cdot e^{i \theta_{4}}-l_{1} e^{i \theta_{1}}=0 \\
P_{H}=l_{a} \cdot e^{i \theta_{a}}+l_{2} \cdot e^{i\left(\theta_{a}+\theta_{2}\right)}+l_{h} \cdot e^{i \theta_{h}}
\end{gathered}
$$

In Eq.(2), $l_{a}$ and $l_{h}$ are the lengths of the lower and upper rigid body vectors. $\theta_{h}$ is hip joint variable and it can be calculated from the geometry.

From Eq.(2), hip position of the artificial leg can be found for the given $\theta_{a}$ and $\theta_{2}$ inputs.

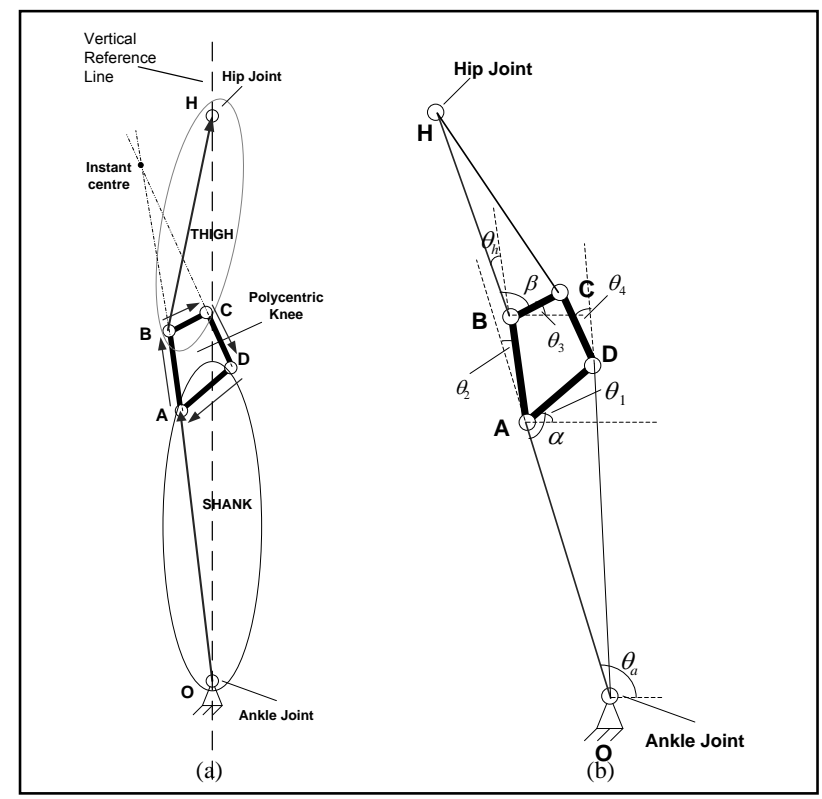

Fig. 1. (a) Artificial hybrid leg model (b) representation of joint variables in flexion mode 


\subsection{Inverse Kinematics Analysis}

Inverse kinematics is the process of determining joint parameters of a kinematic chain in order to achieve a desired pose. Having obtained the workspace, a suitable trajectory within this workspace must be chosen. To obtain the values of joint variable of hybrid artificial knee at every point of the trajectory, iterative solutions must be considered since joint variables of close chain four bar mechanism can only be found by solving nonlinear Eq. (1) and (2) for the given end effector pose.

\subsection{Obtaining Velocity and Acceleration}

The velocity problem in this study is considered as inverse velocity problem in which velocity state of the end effector is given and input joint rates required to produce the desired velocity are found. Velocity equations are given in Eqs. (3) and (4), where $\omega_{t}$ and $\omega_{f}$ are the angular velocities of lower and upper legs, respectively.

$$
\begin{aligned}
& 2 \vec{w}_{t} \times \vec{r}_{H}+\vec{w}_{2} \times \vec{r}_{H / A}+\vec{w}_{f} \times\left(\vec{r}_{H / C}+\vec{r}_{H / B}\right)+\vec{w}_{5} \times \vec{r}_{H / D}=\vec{V}_{H} \\
& \vec{w}_{2} \times \vec{r}_{B / A}+\vec{w}_{f} \times \vec{r}_{C / B}-\vec{w}_{5} \times \vec{r}_{C / D}-\vec{w}_{t} \times \vec{r}_{B / C}=0
\end{aligned}
$$

Acceleration expressions can be obtained, by taking the derivatives of velocity equations with respect to time as giveb in Eqs. (5) and (6):

$$
\begin{aligned}
& 2 \vec{\alpha}_{t} \times \vec{r}_{H}+\vec{\alpha}_{f} \times\left(\vec{r}_{H / C}+\vec{r}_{H / B}\right)+\vec{\alpha}_{2} \times \vec{r}_{H / A}+\vec{\alpha}_{5} \times \vec{r}_{H / D} \\
& =\vec{a}_{H}-2 \vec{w}_{t} \times\left(\vec{w}_{t} \times \vec{r}_{H}\right)-\vec{w}_{f} \times\left[\vec{w}_{f} \times\left(\vec{r}_{H / C}+\vec{r}_{H / B}\right)\right] \\
& -\vec{w}_{5} \times\left(\vec{w}_{5} \times \vec{r}_{H / D}\right)-\vec{w}_{2} \times\left(\vec{w}_{2} \times \vec{r}_{H / A}\right) \\
& \vec{\alpha}_{t} \times \vec{r}_{B / C}+\vec{\alpha}_{2} \times \vec{r}_{B / A}+\vec{\alpha}_{f} \times \vec{r}_{C / B}-\vec{\alpha}_{5} \times \vec{r}_{C / D}= \\
& -\vec{w}_{t} \times\left(\vec{w}_{t} \times \vec{r}_{B / C}\right)-\vec{w}_{2} \times\left(\vec{w}_{2} \times \vec{r}_{B / A}\right)+\vec{w}_{5} \times\left(\vec{w}_{5} \times \vec{r}_{C / D}\right)
\end{aligned}
$$

\section{DYNAMICS OF THE PROSTHETIC LEG}

Three sorts of torques act on the artificial hybrid leg. These are gravity torques, external forces/torques and dynamic torques which arise from the motion of the artificial hybrid leg. Dynamic torques include inertial, centripetal and Coriolis effects. The dynamic equations of hybrid artificial leg cover all these effects and they can be derived by using Newton-Euler formulation.

As mentioned before, motors are going to be mounted on joint $A$ and joint $O$. Hence, torques acting on these joints must be computed. In Figure 2, forces acting on the prosthetic leg are shown. Here, $\tau_{\text {ext }}$ is the external torque exerted to the hip joint, $m_{1}, m_{2}$ and $m_{3}$ are weights of the related links, $f_{e x t}$ is the external force acting at hip joint and $I_{1}, I_{2}$ and $I_{3}$ are the mass moments of inertia of the related links.

For joint $A$, torque demand can be calculated as in Eq.(7). Torque expression $T_{B}$ is given in Eq.(8).

$$
\begin{gathered}
\vec{\tau}_{A}=\vec{\tau}_{B}+\vec{r}_{G_{2} / A} \times m_{2} \cdot \vec{a}_{G_{2}}-\vec{r}_{G_{2} / A} \times m_{2} \cdot \vec{g}+I_{2} \cdot \vec{\alpha}_{2} \\
\vec{\tau}_{B}=\vec{\tau}_{e x t}+\vec{r}_{G_{3} / B} \times m_{3} \cdot \vec{a}_{G_{3}}+\vec{r}_{H / B} \times \vec{f}_{e x t}-\vec{r}_{G_{3} / B} \times m_{3} \cdot \vec{g}+I_{3} \cdot \vec{\alpha}_{f} \\
\vec{\tau}_{O}=\vec{\tau}_{D}+\vec{\tau}_{A}+\vec{r}_{G_{1}} \times m_{1} \cdot \vec{a}_{G_{1}}-\vec{r}_{G_{1}} \times m_{1} \cdot \vec{g}+I_{1} \cdot \vec{\alpha}_{t}
\end{gathered}
$$

As it can be seen from Figure 2, torque expressions $T_{D}$ and $T_{C}$ are calculated as follows:

$$
\begin{gathered}
\vec{\tau}_{D}=\vec{\tau}_{C}+\vec{r}_{G_{4} / D} \times m_{5} \cdot \vec{a}_{G_{4}}-\vec{r}_{G_{4} / D} \times m_{5} \cdot \vec{g}+I_{4} \cdot \vec{\alpha}_{4} \\
\vec{\tau}_{C}=\vec{\tau}_{e x t}+\vec{r}_{G_{3} / C} \times m_{3} \cdot \vec{a}_{G_{3}}+\vec{r}_{H / C} \times \vec{f}_{\text {ext }}-\vec{r}_{G_{3} / C} \times m_{3} \cdot \vec{g}+I_{3} \cdot \vec{\alpha}_{f}(11)
\end{gathered}
$$

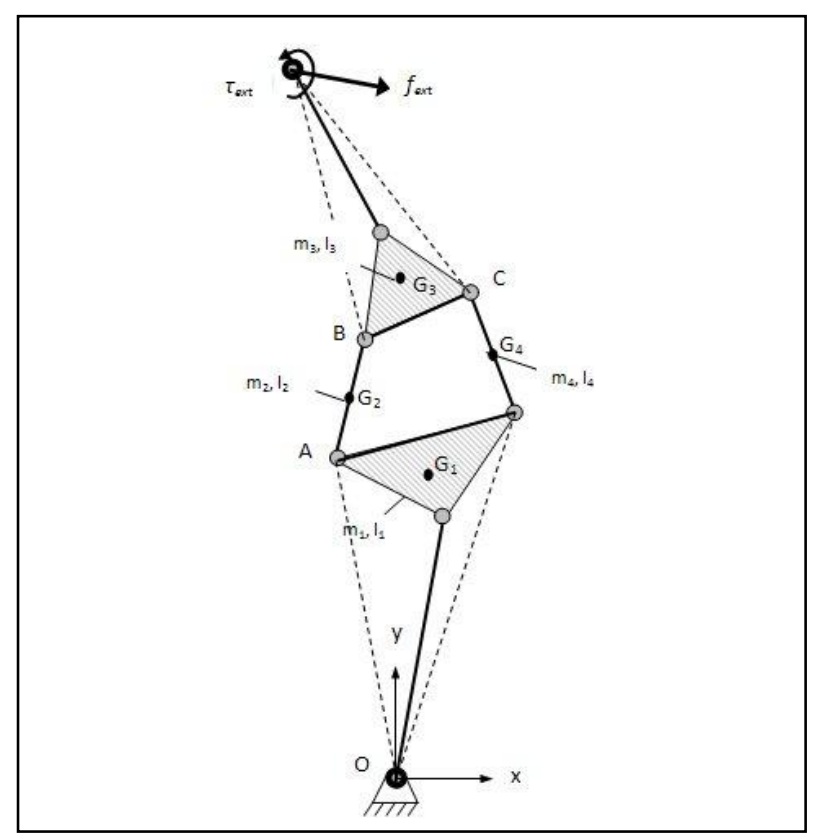

Fig. 2. Representation of acting forces and torques on the hybrid artificial leg

\section{CONCLUSION}

In this study, dynamics of artificial leg is derived in order to calculate the torque demands of the motors which are to be used to actuate the artificial hybrid leg system discussed. As for the knee joint, a polycentric four bar mechanism is chosen. Four bar mechanism has the advantage of changing position of instant centre of rotation by properly choosing the lengths of the anterior and/or posterior links of the four bar mechanism. By doing so, torque demand of the motors can be reduced and smaller motors having lower power rates can be employed. In the studies of Kapti \& Yucenur (2006) it is mentioned that, due to the higher power demand of the prosthetic knee joint motor, total weight of the prosthesis becomes high, which is an undesired property for the artificial leg designs. Hence, lower torque demands of the motor used in the polycentric knee joint mechanism of the artificial hybrid leg leads to a reduction in the total weight of the system. This seems to be an advantage when it is compared with the artificial leg having a single axis knee joint.

In conclusion, it can be said that calculation of the dynamic torques has great importance to predict the torque demands of the motors, since the appropriate motor power can be estimated only after deriving these torque values.

\section{REFERENCES}

Fujimoto, H.; Shimura, Y. \& Kato, I. (1992). Analysis of axial force and moment on above knee prosthesis socket. Journal of Robotics and Mechatronics, 4(4), 268-272.

Kapti, A.O. \& Yucenur, M.S. (2006). Design and Control of an Active Knee Joint. Tribology International, (43), 5-6, 10171026

Michael, J.W. (1999). Modern prosthetic knee mechanisms. Journal of Clinical Orthopedics and Related Researches, $361,39-47$

Radcliffe, C.W. (1994). Four-bar linkage prosthetic knee mechanism: Kinematics, alignment and prescription criteria. Journal of Prosthetics and Orthotics International, $18,159-173$

Pinzur, M.S. \& Bowker, J.H. (1999). Knee disarticulation. Journal of Clinical Orthopedics and Related Researches, $361,23-28$ 